\title{
Formation of oxygenated polycyclic aromatic hydrocarbons from polycyclic aromatic hydrocarbons during aerobic activated sludge treatment and their removal process
}

\author{
Meng Qiao, Weixiao Qi *, Huijuan Liu, Yaohui Bai, Jiuhui Qu \\ Key Laboratory of Drinking Water Science and Technology, Research Center for Eco-Environmental Sciences, Chinese Academy of Sciences, Beijing 100085, China
}

\section{H I G H L I G H T S}

- Transformation from anthracene to anthraquinone was validated in biological treatment.

- Functional fungi for PAH transformation to OPAH were detected in activated sludge.

- The low molecular weight compounds were easier removed than the high ones.

- The middle temperature was fit for compounds removal than the low and high ones.

- The dissolved organic matter probably significantly influenced the compounds removal.

\section{A R T I C L E I N F O}

\section{Article history:}

Received 14 March 2016

Received in revised form 26 April 2016

Accepted 27 April 2016

Available online 27 April 2016

\section{Keywords:}

Oxygenated polycyclic aromatic

hydrocarbons

Polycyclic aromatic hydrocarbons

Biotransformation

Removal

Wastewater treatment plant
G R A P H I C A L A B S T R A C T

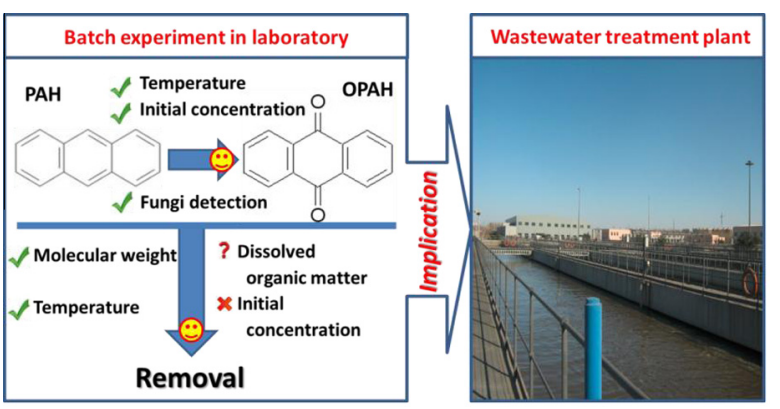

\section{A B S T R A C T}

Though previous studies have focused on the removal of polycyclic aromatic hydrocarbons (PAHs) and their oxygenated derivatives (OPAHs) in wastewater treatment plants, there is a lack of information on the transformation from PAHs to OPAHs during the activated sludge treatment. In this study, a batch experiment was carried out to simulate the aerobic biological treatment process, and the fungi species was detected by high-throughput sequencing. In the result, the transformation from PAHs (anthracene) to OPAHs (anthraquinone) was confirmed. A higher temperature $\left(25^{\circ} \mathrm{C} / 35^{\circ} \mathrm{C}\right.$ ) and higher initial concentration $(5.0 \mu \mathrm{g} / \mathrm{L})$ of anthracene promoted the formation of anthraquinone, compared with a lower temperature $\left(10^{\circ} \mathrm{C}\right)$ and lower initial concentration $(0.5 \mu \mathrm{g} / \mathrm{L})$. The relevant functional fungi for the transformation were detectable in the activated sludge (Candida_parapsilosis, Candida_tropicalis and Bjerkandera-sp), confirming the biotransformation. After $168 \mathrm{~h}$, anthraquinone was totally biodegraded, indicating that activated sludge treatment was satisfactory for PAHs removal from the aspect of the biodegradation of the intermediate OPAHs. Furthermore, the possible factors influencing the biodegradation of both PAHs and OPAHs were also determined. The lower molecular weight compounds were more easily biodegraded than the higher ones. The temperature around $25^{\circ} \mathrm{C}$ was more appropriate for compounds removal than $35^{\circ} \mathrm{C}$ or $10^{\circ} \mathrm{C}$, because the microbial species were more plentiful in activated sludge at $25^{\circ} \mathrm{C}$. Different initial concentrations $(0.5 \mu \mathrm{g} / \mathrm{L}, 5.0 \mu \mathrm{g} / \mathrm{L})$ did not significantly influence the removal efficiency. However, the compounds we added to the samples could be more easily removed than those inherent in the samples, probably resulting from the stronger combination of the inherent compounds with the dissolved organic matters. The results from the batch experiment could be valid indicators for the effect of real wastewater treatment process.

(c) 2016 Elsevier B.V. All rights reserved.

\footnotetext{
* Corresponding author.

E-mail address: wxqi@rcees.ac.cn (W. Qi).
} 


\section{Introduction}

Polycyclic aromatic hydrocarbons (PAHs) and their derivatives, such as oxygenated PAHs (OPAHs), methyl PAHs (MPAHs) and nitrated PAHs (NPAHs) are emitted from incomplete combustions and diesel engines $[1,2]$. The derivatives could also be created by transformation from their corresponding PAHs by chemical and/ or biological processes $[3,4]$. Some of the derivatives are as toxic as or more toxic than their corresponding parent compounds $[5,6]$. Previous studies reported the existence of MPAHs and OPAHs in wastewater treatment plants (WWTPs) as well as in rivers $[7,8]$. Although these compounds can be partially removed in biological WWTPs, the effluent from the corresponding WWTPs is a major source for PAHs and their derivatives in the receiving river systems, especially in Beijing, China [8,9].

It has been reported that PAHs can be transformed to OPAHs by the white rot fungi, which produce the laccase, manganese peroxidase and lignin enzymes. In order to determine the function of a particular white rot fungus, a number of studies investigated the biotransformation from PAHs to OPAHs using a single white rot fungus. Fluorene could be transformed to 9-hydroxyfluorene and 9-fluorenone (the major product) by manganese peroxidase and lignin enzyme [10]. Under the same conditions, anthracene and phenanthrene could transform to 9,10-anthraquinone and then to $\mathrm{CO}_{2}$, and 9,10-phenanthraquinone then to $2,2^{\prime}-$ diformylbiphenyl and finally to $\mathrm{CO}_{2}$, respectively [11]. Vyas et al. investigated various functions of the white rot fungi, and concluded that all these fungi could transform anthracene to anthraquinone [12]. Phanerochaete chrysosporium and strain Px could accumulate anthraquinone, and Pleurotus ostreatus, Coriolopsis polyzona and Trametes versicolor could further degrade anthraquinone, while $P$. ostreatus and $C$. polyzona could not degrade anthraquinone in the presence of anthracene [13]. There are still some studies reported the formation of anthraquinone from anthracene in real soil environment by the function of white rot fungi [4]. However, there has been few study focused on the performance of activated sludge in transforming PAHs to OPAHs. The formation of OPAHs in the wastewater treatment process has never been reported before, except a speculative conclusion in our previous study [7]. Meanwhile, whether the white rot fungi exist in WWTPs is also unknown.

The removal process of PAHs in WWTPs has been well investigated. In the biological combined with chemical precipitation treatment process, the removal efficiencies of $\Sigma 16$ PAHs (94$100 \%$ ) were higher than those in the mechanical and the chemical combined mechanical process (25-78\%) [14]. The influence of the molecular weight and temperature on the removal of PAHs in WWTPs has also been considered. Generally speaking, compared to the higher molecular weight (HMW) PAHs, the removal efficiencies of the lower molecular weight (LMW) PAHs were much higher, because the LMW PAHs could be more easily biodegraded/biotransformed than the HMW PAHs [15]. Combined with different treatment process, the HMW PAHs were easier to remove by chemical precipitation, while the LMW PAHs were necessary to remove by biological treatment [14]. The removal efficiencies of PAHs varied in different seasons with different temperatures. In summer, the removal efficiencies of PAHs were higher than those in winter, due to a more appropriate temperature in summer for microorganisms [16]. Regarding to SPAHs, only a few studies have reported the removal of MPAHs and OPAHs in WWTPs. Pham and Proulx investigated the concentrations of MPAHs both in the influent and effluent from a WWTP using physical-chemical treatment [17]. The average concentrations of 5 individual MPAHs in the influent ranged from 53 to $94 \mathrm{ng} / \mathrm{L}$, and in the effluent from 15 to $35 \mathrm{ng} / \mathrm{L}$; the removal efficiencies for individual MPAHs were between 53\% and 75\%. Berqvist et al. examined the removal efficiencies of 5 most abundant MPAHs [15]. The concentrations of the individual naphthalene derivatives were $21-130 \mathrm{ng} / \mathrm{L}$ in the influent and 1-27 ng/L in the effluent. For other PAH derivatives, to the best of our knowledge, only one study from our group reported the removal of OPAHs in a biological WWTP [7]. Thus, there is a lack of study focused on the removal process and influencing factors of SPAHs. Although a number of study reported the behavior of PAHs in WWTPs, the process in real WWTPs would be influenced by varies environmental factors. Accordingly, the simulation of a batch experiment is indicated to be necessary, and it will be studied.

Therefore, the aims of this study were to: (1) validate the transformation from PAHs to OPAHs; (2) figure out the definite influencing factor for the biodegradation/biotransformation of PAHs and OPAHs; (3) compare the behavior of the compounds in a real WWTP and the batch experiment, to make the results from the batch experiment as indicators for the treatment effect of the real WWTPs.

\section{Materials and methods}

\subsection{Chemicals}

Six d-PAHs, including d-fluorene (d-Fluo, $10 \mu \mathrm{g} / \mathrm{mL}$ ), dphenanthrene (d-Phe, $200 \mu \mathrm{g} / \mathrm{mL}$ ), d-anthracene (d-Ant, $2000 \mu \mathrm{g} /$ $\mathrm{mL}$ ), d-pyrene (d-Pyr, $500 \mu \mathrm{g} / \mathrm{mL}$ ), d-benz[a]anthracene (d-BaA, $2000 \mu \mathrm{g} / \mathrm{mL}$ ) and d-benzo[a]pyrene (d-BaP, $10 \mu \mathrm{g} / \mathrm{mL}$ ); one d$\mathrm{OPAH}$, d-anthraquinone (d-AQ, $100 \mu \mathrm{g} / \mathrm{mL}$ ); three OPAHs, encompassing 9-fluorenone (9-FL, in solid $100 \%$ ), anthraquinone (AQ $100 \mu \mathrm{g} / \mathrm{mL}$ ), benz[a]anthracene-7,12-dione (BA-7,12-D, $50 \mu \mathrm{g} /$ $\mathrm{mL}$ ); and the sixteen USEPA priority PAHs, including naphthalene (Nap), acenaphthylene (Acy), acenaphthene (Ace), fluorene (Fluo), phenanthrene (Phe), anthracene (Ant), fluoranthene (Flua), pyrene (Pyr), benz[a]anthracene (BaA), chrysene (Chry), benzo[b]fluoranthene $(\mathrm{BbF})$, benzo[k]fluoranthene $(\mathrm{BkF})$, benzo[a]pyrene $(\mathrm{BaP})$, indeno[1,2,3-cd]pyrene (IcdP), dibenz[a,h]anthracene (DBA), and benzo[g,h,i]perylene (BghiP), in a mixture $(200 \mu \mathrm{g} / \mathrm{mL})$, were purchased from AccuStandard, Inc., New Haven, USA. Internal standards for 2-fluorobiphenyl (2-FB, in solid >96\%) and decachlorobiphenyl (PCB209) were obtained from Aldrich Chemical Co., Inc. (Gillingham, Dorset, UK).

\subsection{Biodegradation and transformation test system}

The influent and activated sludge were collected from Qinghe WWTP in Beijing, China, in April, 2014. After transport to the laboratory, the influent and activated sludge were mixed in a 1:1 ratio, as in the WWTP. The average suspended solid volume (SSV) in each incubator was $4069 \pm 368 \mathrm{mg} / \mathrm{L}$.

The experiment simulated three temperature conditions, low temperature $\left(10^{\circ} \mathrm{C}, \mathrm{LT}\right)$, middle temperature $\left(25^{\circ} \mathrm{C}\right.$, MT) and high temperature $\left(35^{\circ} \mathrm{C}, \mathrm{HT}\right)$. Every sample was acclimated for 3 days, in aerobic conditions. The acclimation experiment was conducted in biochemical incubators, at constant temperature and in darkness. After the pretreatment, $0.5 \mu \mathrm{g} / \mathrm{L}$ d-PAHs (d-Fluo, d-Phe, d-Ant, d-Pyr, d-BaA, d-BaP) were spiked into each sample.

To determine the influence of the initial PAH concentrations, $5.0 \mu \mathrm{g} / \mathrm{L}$ of 4 types of d-PAH (d-Phe, d-Ant, d-Pyr, d-BaA) were added at $25{ }^{\circ} \mathrm{C}$. In addition, for investigating the removal process of the 16 PAHs and 3 OPAHs, at the same temperature and the same initial d-PAH concentrations, certain amounts of the 3 OPAHs $(5.0 \mu \mathrm{g} / \mathrm{L})$ and 16 PAHs $(5.0 \mu \mathrm{g} / \mathrm{L})$ were added. Thus, in the same 
time, the removal processes for d-PAHs could be performed in duplicate to validate the stability of the biological process. The measured concentrations of the added PAHs and OPAHs were calculated as the total concentrations of the inherent and added targets in the control experiment minus the concentrations of the inherent targets in the experiment.

The samples were collected in a 7-day period between $0 \mathrm{~h}$ and $168 \mathrm{~h}$, at 6 time intervals $(0 \mathrm{~h}, 8 \mathrm{~h}, 24 \mathrm{~h}, 48 \mathrm{~h}, 96 \mathrm{~h}$, and $168 \mathrm{~h}$ ). $\mathrm{NaN}_{3}$ was added immediately after the samples were collected to inhibit further biodegradation. The aqueous phase and solid phase were separated by centrifugation and filtration. The aqueous phase samples were extracted within one day. The solid phase samples were stored in a refrigerator below $-20^{\circ} \mathrm{C}$ for chemical analysis, and below $-80^{\circ} \mathrm{C}$ for biological analysis until use.

\subsection{Chemical analytical methods}

The analytical procedure has been reported by Qiao et al. [18]. A brief description of the method is listed below. Aqueous phase samples were extracted using solid phase extraction (SPE) with C18 cartridges ( $500 \mathrm{mg}, 6 \mathrm{ml}$, Supelco). Solid phase samples were extracted with accelerated solvent extraction (ASE300, Donex). After solvent exchange and concentration, samples were purified with silica gel/alumina cartridges. Elution fractions were concentrated to $0.5 \mathrm{~mL}$ under a gentle stream of nitrogen. The internal standards (100 ng) were added to each sample before instrumental analysis.

The targets were detected by an Agilent 7890A GC equipped with a 5795C mass detector (GC-MS). A DB-17MS fused silica capillary column $(30 \mathrm{~m} \times 0.25 \mathrm{~mm} \times 0.25 \mu \mathrm{m})$ was used for separation of the compounds. Samples $(1 \mu \mathrm{L})$ were injected in splitless mode. The carrier gas was helium at a rate of $1 \mathrm{~mL} / \mathrm{min}$. The program procedure for SPAHs and PAHs was as follows: from $60^{\circ} \mathrm{C}$ (hold $1 \mathrm{~min}$ ) to $110^{\circ} \mathrm{C}$ at a rate of $20^{\circ} \mathrm{C} / \mathrm{min}$, and to $290^{\circ} \mathrm{C}$ at $3^{\circ} \mathrm{C} / \mathrm{min}$ (hold $20 \mathrm{~min}$ ).

The details of the compounds recoveries and the method quantification limits (MQLs) were shown in Supporting information (Table S1).

\subsection{Biological analytical method}

The total DNA was extracted from the sludge samples using PowerSoil DNA Isolation Kit (Mobio, USA) according to the manufacturer's instruction. Each sample was performed in triplicate. The DNA samples were stored at $20^{\circ} \mathrm{C}$ until analysis.

The DNA samples were sent to BGI (Beijing Genomics Institute) for PE250 paired-end sequencing on the platform of Illumina MiSeq (ITS2: forward primer: GCATCGATGAAGAACGCAGC; reverse primer: TCCTCCGCTTATTGATATGC) to identify the composition of the fungal community in the activated sludge. The raw data of MiSeq sequencing were treated and analyzed as follows: (1) data filtration. The raw sequences reads were initially filtered to remove low quality reads, barcode primers and ambiguous sequences. (2) Paired-end reads were connected to the tags. If the two paired-end reads overlapped, the consensus sequence was generated by FLASH (Fast Length Adjustment of Short reads, v1.2.11). The paired-end reads without overlaps were removed. In average, 82825 tags per sample were obtained and the length is roughly 299 bps. (3) Species annotation. The tags were clustered to OTU (Operational Taxonomic Unit) by $97 \%$ similarity by software USEARCH (v7.0.1090). The species were identified according to blast against Unite database [19]. Species histogram was plotted using the software R (v3.0.3) [20].

\section{Results and discussion}

\subsection{Biotransformation from $P A H$ to $O P A H$}

The removal of PAHs during a biological treatment was not only by biodegradation, but also by biotransformation. We proposed in the previous study that in a real biological WWTP, some PAHs could transform to OPAHs [7,8]. In this section, one of the intermediate products of $\mathrm{d}-\mathrm{Ant}, \mathrm{d}-\mathrm{AQ}$, was investigated to confirm the biotransformation from PAHs to OPAHs during biological wastewater treatment process. In addition, the fungi in the activated sludge were determined.

\subsubsection{Formation of $A Q$ from Ant}

The mass of d-AQ was determined after d-Ant was added (Fig. 1). D-AQ was immediately formed under different conditions at $0 \mathrm{~h}$. It could be concluded from Fig. 1 that different temperatures and different initial d-Ant concentrations influenced the formation concentrations of d-AQ. Finally, at $168 \mathrm{~h}$, all d-AQ totally disappeared.

The initial concentration of d-Ant was $0.5 \mu \mathrm{g} / \mathrm{L}$ in experiments to determine the influence of temperature (Fig. 1a). In the HT condition, the highest d-AQ concentration occurred at $0 \mathrm{~h}$, while the in MT condition at $48 \mathrm{~h}$. The highest concentration of d-AQ in the HT $(0.05 \mu \mathrm{g} / \mathrm{L})$ and MT $(0.05 \mu \mathrm{g} / \mathrm{L})$ condition were higher than in the LT $(0.03 \mu \mathrm{g} / \mathrm{L})$ condition. The result indicated that the MT and HT condition was more suitable for the formation of $\mathrm{d}-\mathrm{AQ}$ than the LT conditions, but the process in the HT and MT were different. The removal molar concentrations of d-Ant at 0 h were $0.9 \times 10^{-6}$ $\mathrm{mol} / \mathrm{L}, 0.9 \times 10^{-6} \mathrm{~mol} / \mathrm{L}$ and $0.7 \times 10^{-6} \mathrm{~mol} / \mathrm{L}$ in the HT, MT and LT conditions, respectively. The highest molar concentrations of d-AQ formed were $0.2 \times 10^{-6} \mathrm{~mol} / \mathrm{L}, 0.2 \times 10^{-6} \mathrm{~mol} / \mathrm{L}$ and $0.1 \times 10^{-6}$ $\mathrm{mol} / \mathrm{L}$. As a result, the loss amount of d-Ant was higher than the amount of d-AQ formed, probably because the amount of fungi that functioned to biotransform Ant to AQ was low in the activated sludge. Some other compounds might be formed, but not focused in this study.

In the same temperature condition (MT), the higher initial concentration of d-Ant was added, the higher concentration of d-AQ was formed (Fig. 1b). When the added concentration of d-Ant was $0.5 \mu \mathrm{g} / \mathrm{L}\left(2.7 \times 10^{-6} \mathrm{~mol} / \mathrm{L}\right)$, the maximum concentration of $\mathrm{d}$-AQ formed was $0.05 \mu \mathrm{g} / \mathrm{L}\left(0.2 \times 10^{-6} \mathrm{~mol} / \mathrm{L}\right)$; while when the added concentration of $\mathrm{d}$-Ant was $5.0 \mu \mathrm{g} / \mathrm{L}\left(27 \times 10^{-6} \mathrm{~mol} / \mathrm{L}\right)$, the concentration of $\mathrm{d}$-AQ formed was $0.3 \mu \mathrm{g} / \mathrm{L}\left(1.6 \times 10^{-6} \mathrm{~mol} / \mathrm{L}\right)$.

Meulenberg et al. studied the biodegradability of intermediate products of PAH oxidation, and found that the product AQ was more biodegradable than the parent Ant [21]. So, the biodegradation process of AQ should be fitted for the exponential decay. However, the removal process of $\mathrm{d}-\mathrm{AQ}$ did not fit well $\left(R^{2}\right.$ ranged from 0.4556 to 0.9537 ), suggesting that the biodegradation was not the only process during d-AQ removal (Fig. 1). The concentrations of dAQ first increased or slightly decreased during the process, indicating the consistent formation of d-AQ before d-Ant was totally biodegraded.

\subsubsection{The fungi in the biological treatment process}

Due to a better transformation in the MT and HT condition, the species of fungi in the activated sludge in the HT and MT conditions were determined during the 7-day treatment process. It has been reported that the fungi Candida_parapsilosis, Candida_tropicalis and Bjerkandera-sp had the ability to transform PAH to OPAH [22]. The relative abundance of the 3 species of fungi ranged from $0 \%$ to $9 \%$ during the PAH removal process (Fig. S1). Compared to other species, the absolute abundances of these fungi were not very high (Table S2). Thus, the formation amount of AQ was not 


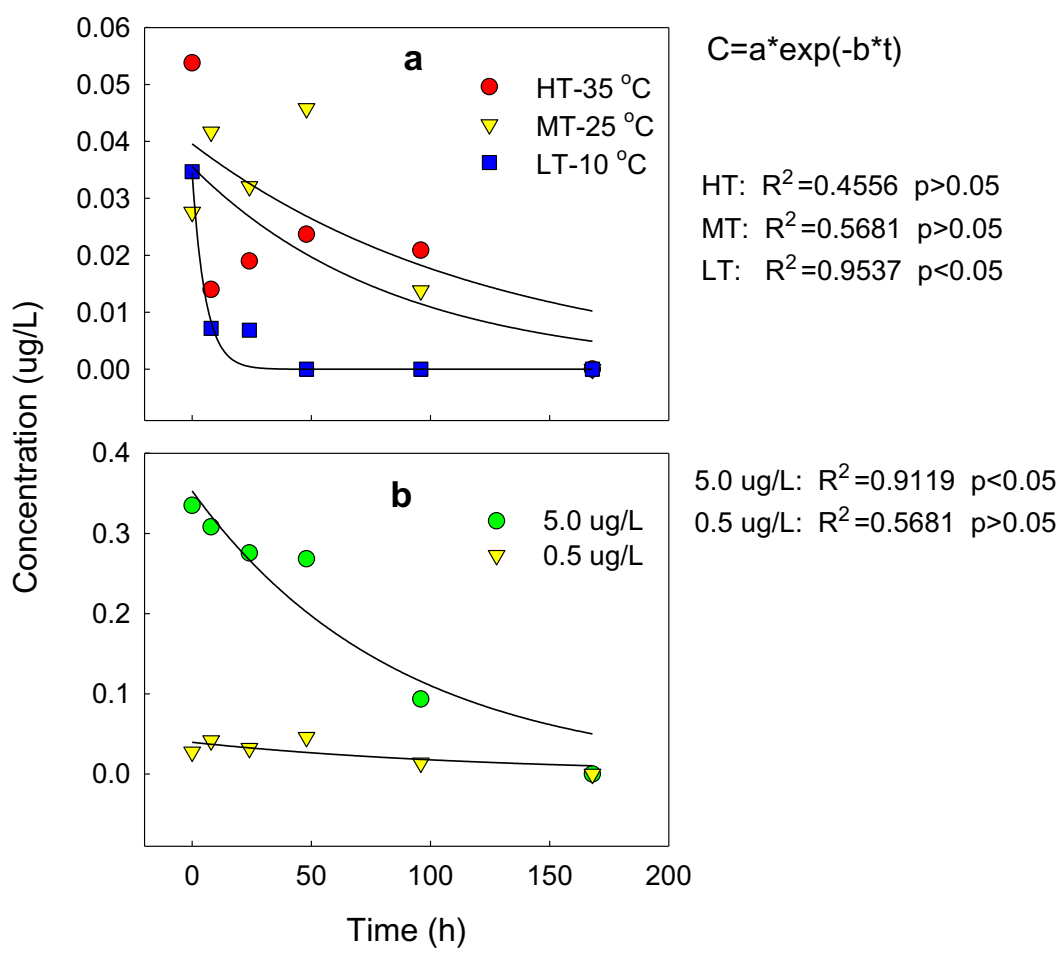

Fig. 1. Formation and biodegradation of d-AQ under different conditions: (a) initial concentration of d-Ant $0.5 \mu \mathrm{g} / \mathrm{L}$; (b) in middle temperature condition.

high, correspondingly. The identification of fungi in the activated sludge was given powerful evidence for the occurrence of the transformation.

Consequently, the transformation from $\mathrm{PAH}$ to OPAH was validated through the above experiments. Finally, all the formed OPAH would be totally removed both in the total phase, indicating that the activated sludge treatment was satisfactory for PAHs removal from the aspect of the biodegradation of the intermediate OPAHs.

\subsection{Removal of PAHs and OPAHs}

Both the PAHs and OPAHs would be biodegraded/biotransformed during the aerobic activated sludge treatment. The removal processes of PAHs and OPAHs in the aqueous phase and the total phase (both the aqueous and solid phase) will be discussed in this section. The same concentrations of d-PAHs during the biological process were performed in duplicates. The relative standard deviations (RSDs) were below 10\% in the aqueous phase and below $20 \%$ in the solid phase.

\subsubsection{The influence of PAH and OPAH molecular weight on the removal kinetics}

D-Fluo, d-Phe, d-Ant, d-Pry, d-BaA and d-BaP were taken as the typical compounds to examine the molecular weight (from 2 rings to 5 rings) influence of PAHs removal. Fluo, Phe, Ant, Pry, BaA, BaP were used for comparison to the relevant d-compounds. 9-FL, AQ and BA-7,12-D were taken as the typical compounds for examining the molecular weight influencing of OPAHs removal.

After the deuterium-substituted (d-Fluo, d-Phe, d-Ant, d-Pry, d$\mathrm{BaA}$ and d-BaP) and target compounds (Fluo, Phe, Ant, Pry, BaA, BaP, 9-FL, AQ and BA-7,12-D) were added to the mixture of the aqueous and solid phase, some of them immediately disappeared in the aqueous phase, especially the higher molecular weight compounds (Fig. 2). Generally speaking, the residues of the 6 typical dPAHs and PAHs at $0 \mathrm{~h}$ in the aqueous phase were less than $10 \%$ of the initial concentrations, and the residues of OPAHs were less than $60 \%$. The higher the ring number of the compounds, the less partitioned in the aqueous phase. In the total phase, the residues of these compounds at $0 \mathrm{~h}$ ranged from $40 \%$ to $99 \%$. The result indicated that PAHs and OPAHs firstly underwent an adsorption process. The $\log K_{\text {ow }}$ value of PAHs increased with the increasing PAH molecular weight [23]. Compounds with a higher $\log K_{\text {ow }}$ value were adsorbed more easily on the solid phase.

The removal process of PAHs (d-PAHs) in the aqueous phase from $0 \mathrm{~h}$ to $168 \mathrm{~h}$ fit well for the first-order reaction, with the correlation coefficients $\left(R^{2}\right)$ between 0.9982 and 1.0000 . It was also reported previously that the biodegradation of Nap, Ant, Phe and Pyr fitted for the first-order kinetics [24-26], met the result in this study. The reaction rate constant $-b$ stands for speed of the chemical reaction, suggesting a lower stability of the compounds with a higher $b$ value. The removal rate of PAHs (d-PAHs) in the aqueous phase slightly decreased and then increased with the increasing molecular weight. The biodegradation rate of PAHs should decrease with the increasing molecular weight. And the adsorption rate increased with the increasing molecular weight. Therefore, the result implied that the residue compounds in the aqueous phase were removed both by biodegradation and adsorption. The residues of 9-FL and AQ at $0 \mathrm{~h}$ were much higher than the other PAHs and OPAHs. And the removal process for AQ and BA-7,12-D did not fit well for the first-order reaction, with $R^{2}=0.9822$ and 0.8221 . The difference of OPAHs with PAHs (d-PAHs) suggested again the possible formation of OPAHs from PAHs.

In the total phase samples, the compounds were mainly removed by biodegradation and biotransformation. The biodegra dation/biotransformation process started as soon as the compounds entered into the mixture of water and sludge. The biodegra dation/biotransformation process of the 2-ring and 3-ring PAHs (dPAHs) fit well with the first-order reaction, with $R^{2}$ between 0.9932 and 1.0000. The $R^{2}$ for 4-ring and 5-ring compounds were a little lower, ranged from 0.9663 to 0.9804 . While the $R^{2}$ for the 6 -ring compound were relatively low, 0.5927 and 0.6259 . The $p$ values for each model were shown in Fig. 2. The confidence intervals for 

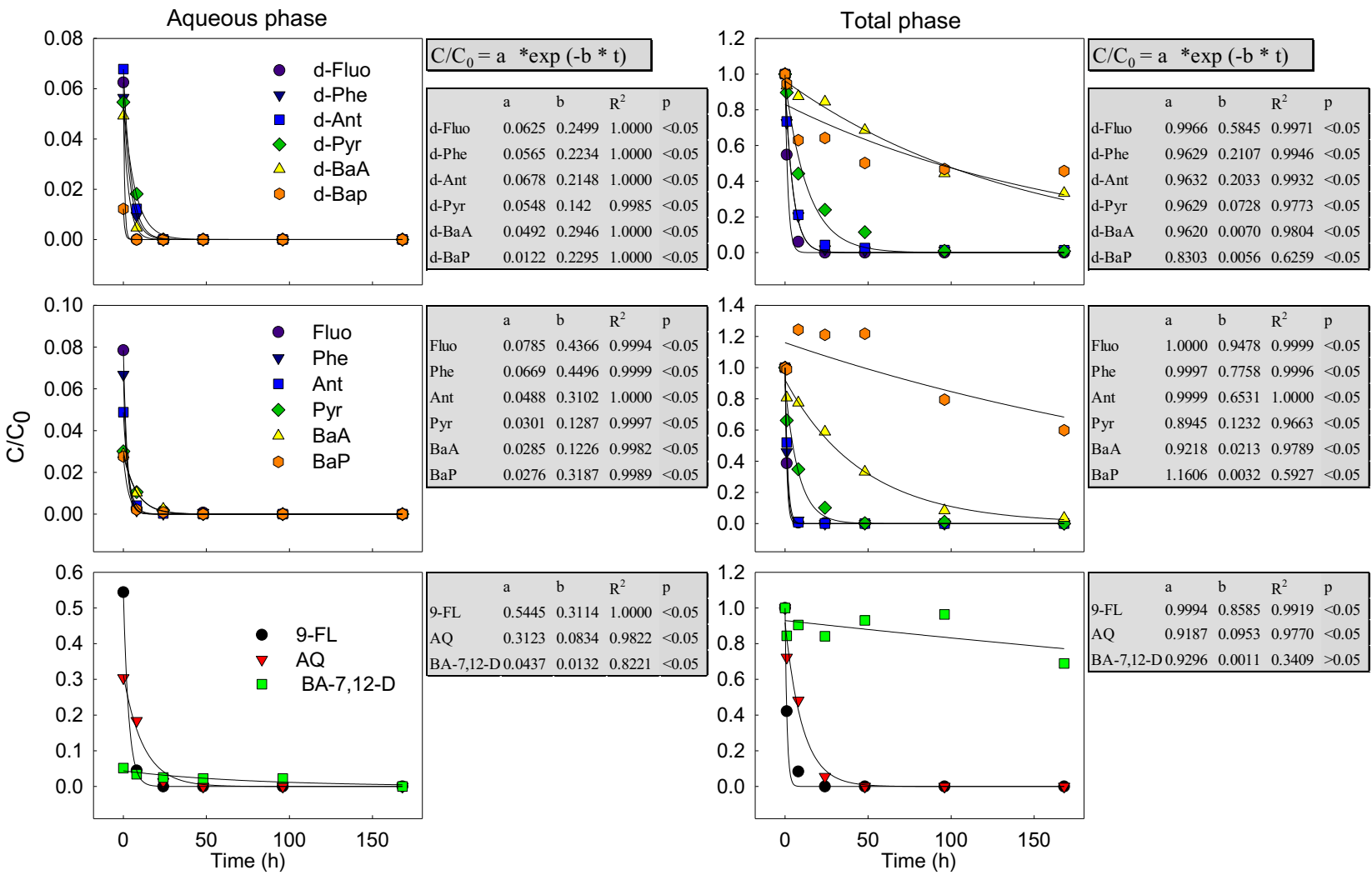

Fig. 2. Removal dynamic of d-PAHs, PAHs and OPAHs in the aqueous and total phase.

PAHs and most OPAHs (except BA-7,12-D in the total phase) were above $95 \%$. The results showed that as the growing of the molecular weight, the biodegradation/biotransformation process was not only influenced by the nature of the target, but also by other extrinsic environmental factors. Based on the constant $-b$ value, the influence of the molecular weight on the biodegradation/bio transformation rate of PAHs and OPAHs in the total phase was significant. As the increasing of the molecular weight, the removal rate decreased. The same phenomenon occurred for OPAHs.

The removal processes for most compounds reached balance both in aqueous and total phase at $168 \mathrm{~h}$. The removal efficiencies of the added PAHs and OPAHs at $168 \mathrm{~h}$ from the aqueous phase ranged from $96 \%$ to $100 \%$ for the 16 PAHs and $100 \%$ for the 3 OPAHs. From the total phase, PAHs with lower molecular weight ranging from 128 to 228 (2-4 rings) were removed 88$100 \%$, while those with higher molecular weight from 252 to 278 (4-6 rings) decreased 11-40\%; for OPAHs, the removal efficiencies of $9-\mathrm{FL}$ and AQ were $100 \%$ and $99 \%$ respectively, but BA7,12-D showed only $31 \%$ removal efficiency (Fig. S2). To understand the relationship between the removal efficiency and the molecular weight, the linear correlation factors were calculated (Fig. S3). The removal efficiencies of the added targets in the total phase decreased with increasing molecular weight, with a corresponding factor of 0.76 for PAHs and 0.88 for OPAHs. Similarly, in a real WWTP, the reduction of LMW PAHs and OPAHs in the total phase was higher than the reduction of HMW PAHs and OPAHs [7]. In the aqueous phase, the situation was different. The removal efficiencies showed less dependence on the molecular weight, with a much lower corresponding factor of 0.38 for PAHs and 0.60 for OPAHs. The removal efficiencies of the PAHs and OPAHs in the aqueous phase did not vary much with the molecular weight, which will be further discussed in Section 3.2.4.

\subsubsection{The influence of temperature on PAHs removal}

As discussed in the above section, most of the target compounds partitioned in the solid phase as soon as they were added to the mixture. The aqueous phase contained $0-16.1 \%$ of the target compounds, while the solid phase contained $83.9-100 \%$. The partitioning of the compounds in the aqueous phase in the LT condition were the highest among the 3 temperature conditions, followed by the HT, and then the MT (Fig. S4). The result showed that the adsorption rates of the compounds onto the solid phase were the fastest in the MT condition, followed by the HT, and the slowest in the LT condition, which would influence the removal process of PAHs and OPAHs in the aqueous and total phase.

For the aqueous phase samples (Fig. 3 top), the removal efficiency of d-Fluo was 100\% in the 3 temperature conditions at $8 \mathrm{~h}$. The removal rates of $\mathrm{d}$-Phe, d-Ant and d-Pyr in the LT condition were slower than those in the HT and MT conditions. The removal efficiency of d-Phe reached 100\% in the MT condition using at $8 \mathrm{~h}$, while the $100 \%$ removal in the HT and LT conditions required $24 \mathrm{~h}$. The d-Ant was totally removed in the MT and HT conditions within $8 \mathrm{~h}$, while in the LT conditions $48 \mathrm{~h}$ was required. Similarly, the removal efficiency of d-Pyr was $100 \%$ in the MT and HT conditions within $24 \mathrm{~h}$, and in the LT condition within $48 \mathrm{~h}$. However, d-BaA and $\mathrm{d}-\mathrm{BaP}$ could not be detected at $0 \mathrm{~h}$ in the MT and HT conditions, and at $8 \mathrm{~h}$ in the LT and MT conditions. Accordingly, the removal rate in the aqueous phase samples was the slowest in the LT condition, and in the HT condition it was a little slower than in the MT condition.

For the total phase samples (Fig. 3 bottom), the influence of different temperatures became more significant with the increasing molecular weight. There was little difference in the removal rates of d-Fluo, d-Ant and d-Phe among the 3 different temperatures. The removal rates were a little slower in the LT condition, and relatively the same between the MT and HT conditions. For 

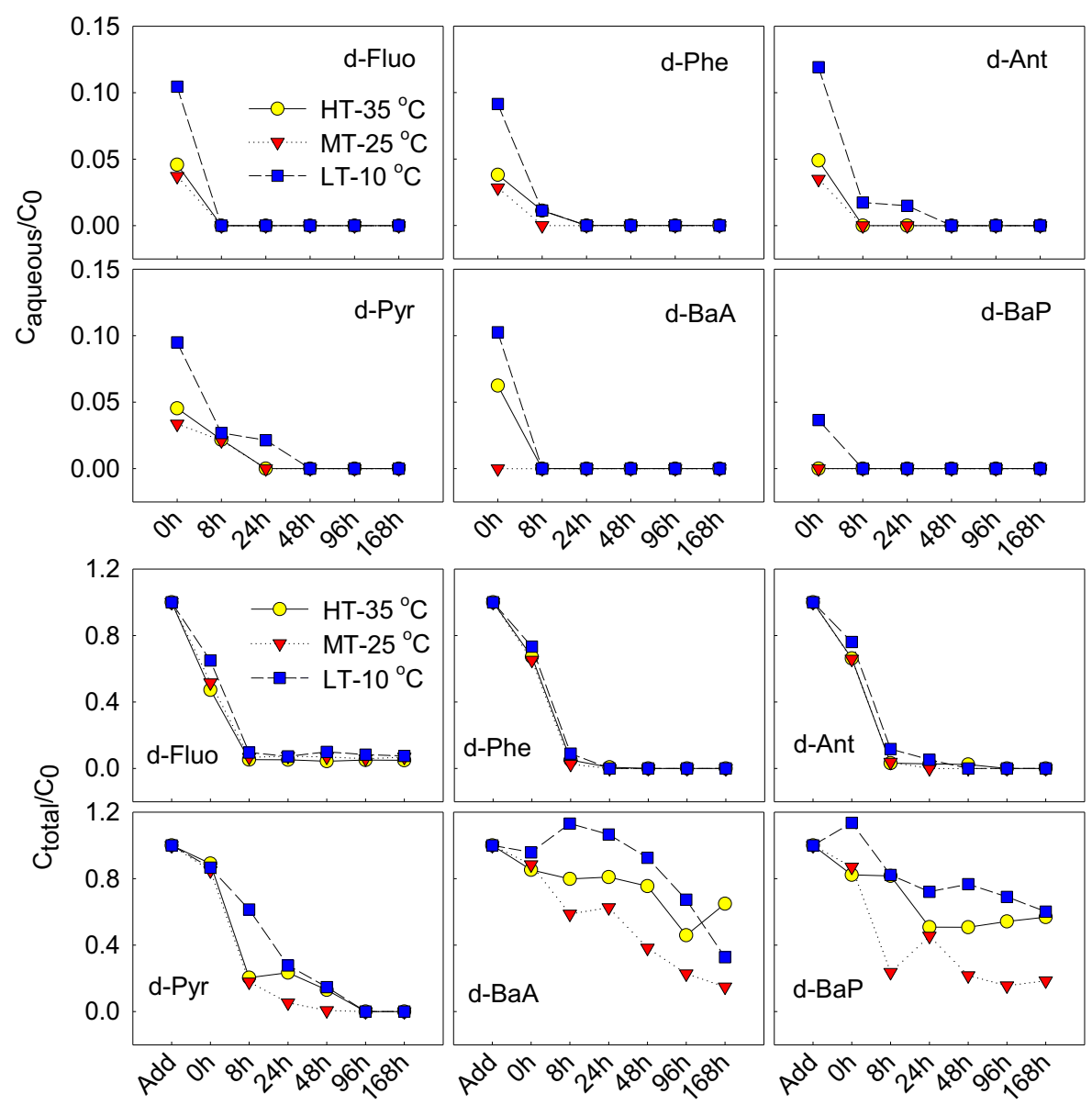

Fig. 3. Residues of d-PAHs in the aqueous phase and total phase in different temperature conditions.

d-Pyr, d-BaA and d-BaP, the removal rates varied with the 3 conditions. The figure showed that the removal rates were the slowest in the LT condition, followed by the HT, and fastest in the MT condition. This phenomenon was the same as observed for the influence of temperature on partitioning. It was reported in a previous reference that $25^{\circ} \mathrm{C}$ to $30^{\circ} \mathrm{C}$ was the most favorable temperature condition for the removal of COD by activated sludge treatment. The removal efficiency would be decreased with both the increasing and decreasing of temperature. The results of microscopy demonstrated that the microbial species were the most plentiful in activated sludge at $25-30^{\circ} \mathrm{C}$, but significantly decreased above $35^{\circ} \mathrm{C}$ [27]. Therefore, MT $\left(25^{\circ} \mathrm{C}\right)$ was the most appropriate temperature for PAH removal by activated sludge treatment.

\subsubsection{The influence of initial concentrations on PAHs removal}

To determine the effect of the initial concentrations on the $\mathrm{PAH}$ removal, we selected 4 d-PAHs (d-Phe, d-Ant, d-Pyr and d-BaA). For the aqueous phase samples, the final removal efficiencies of $d$ PAHs with the initial concentrations of $0.5 \mu \mathrm{g} / \mathrm{L}$ were all $100 \%$; while those with the initial concentrations of $5.0 \mu \mathrm{g} / \mathrm{L}$ ranged from $93 \%$ to $97 \%$ (Fig. 4). The same phenomenon occurred in the total phase. The final removal efficiencies with the lower initial concentration ranged from $83 \%$ to $99 \%$, and at the higher initial concentration they ranged between $93 \%$ and $97 \%$. As a result, the removal efficiencies at the lower initial concentration were a bit higher than those at the higher initial concentration in the aqueous phase, but not obvious, suggesting that the microorganism for PAH degradation and the adsorption capacity of the sludge for PAHs were sufficient with these initial concentrations.
The initial concentrations of the inherent PAHs and OPAHs after the 3-day acclimation before adding the target compounds are listed in Table S3, ranging from $0.10 \mu \mathrm{g} / \mathrm{L}$ to $4.63 \mu \mathrm{g} / \mathrm{L}$ in the total phase for individual targets. The removal efficiencies ranged from $0 \%$ to $56 \%$. With relatively the same initial concentrations as the added compound, the removal efficiencies were much lower. Meanwhile, the corresponding factor between the initial concentration and the removal efficiencies was only 0.07 (Fig. S5). Accordingly, the influence of the initial concentration on the removal efficiencies was not significant.

\subsubsection{The influence of the inherent and added compounds on PAHs removal}

For the removal of PAHs and OPAHs, we found that the final removal efficiencies of the inherent PAHs and OPAHs were different from those of the added ones. It can be seen from Fig. 5 that in the aqueous phase samples, the added PAHs and OPAHs were removed $96-100 \%$, while the inherent ones were only decreased by $9-41 \%$. For the total phase, the final removal efficiencies of the added PAHs and OPAHs (11-100\%) were also higher than the inherent ones (0-28\%). The inherent compounds had been combined with the dissolved organic matters (DOMs) for at least 3 days or more. While the added compounds were not contact with the DOMs before the beginning of the removal process. It has been reported that the DOMs in the aqueous phase might prevent the inherent PAHs and OPAHs from adsorbing on the sludge [28]. As discussed in Section 3.2.1 that these compounds were first adsorbed on the sludge, then continued the adsorption and began the biodegradation/biotransformation. Therefore, the DOMs might 


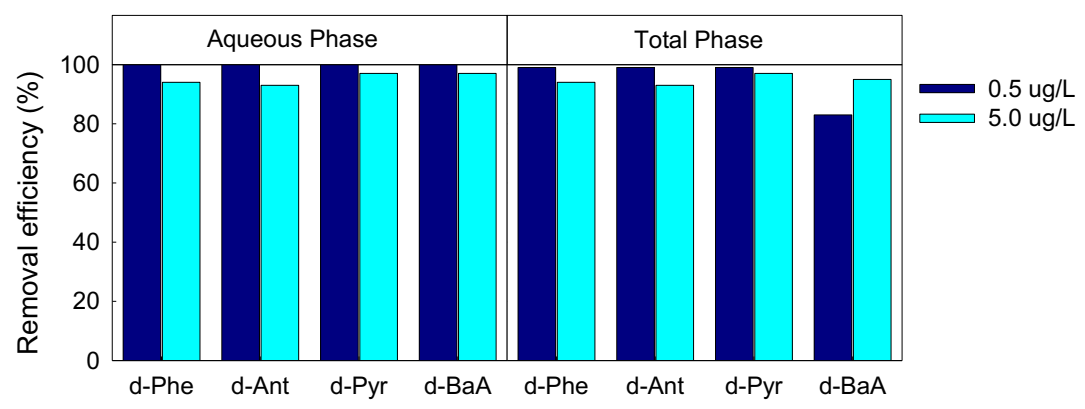

Fig. 4. Removal efficiencies of d-PAHs in aqueous and total phase at different initial concentrations.

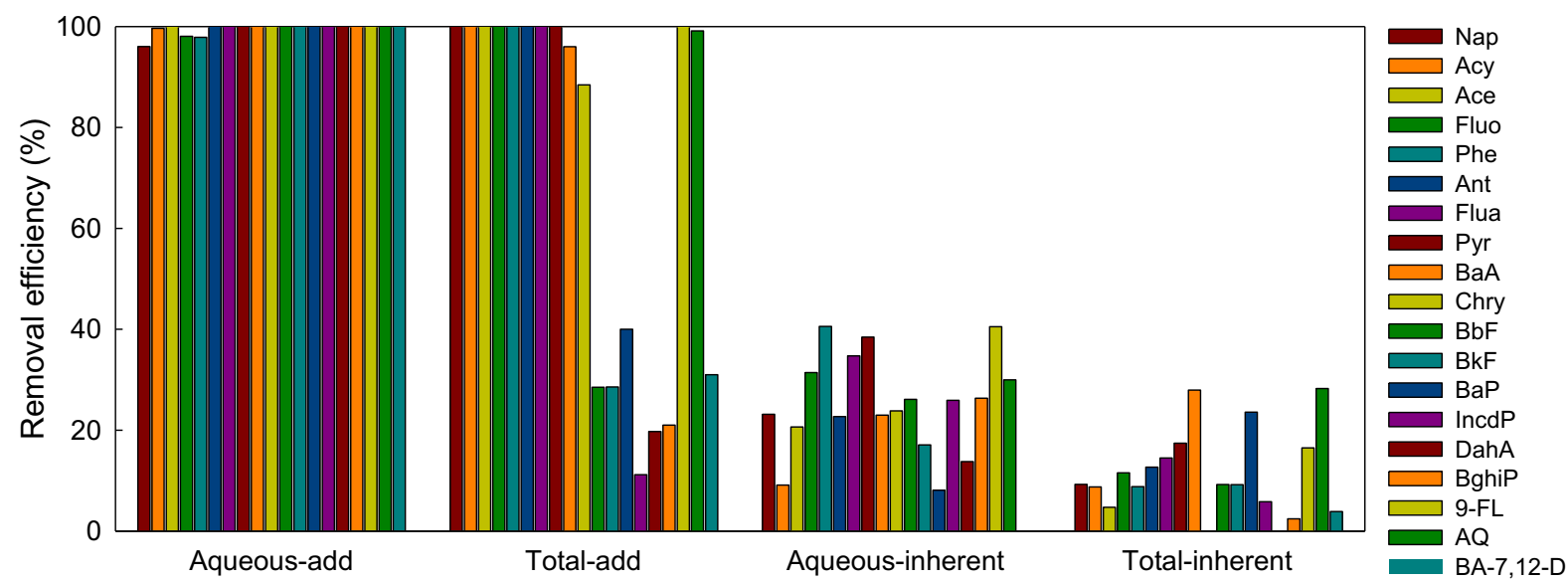

Fig. 5. Removal efficiencies of the added and inherent PAHs and OPAHs in the aqueous and total phase.

decrease both the adsorption and biodegradation/biotransforma tion rates of PAHs and OPAHs in the aqueous and total phase.

\subsection{Comparison of the PAHs/OPAHs behavior between the batch experiment and a real WWTP, and implications}

In the total phase of the influent in a real WWTP, the concentrations of the individual PAHs and OPAHs ranged from $0.04 \mu \mathrm{g} / \mathrm{L}$ to $1.7 \mu \mathrm{g} / \mathrm{L}$ [7]. The SSV in the real WWTP was $4176 \pm 157 \mathrm{mg} / \mathrm{L}$. Both the initial concentrations of the individual compound and the SSV in this batch experiment were designed according to those in the real WWTP.

The batch experiment validated the formation of OPAHs from PAHs in a biological treatment process, which was speculated in the real WWTP [7]. OPAHs were totally removed after a 7-day treatment. However, in the real WWTP, OPAHs was considered to be accumulated, which should be due to the sustainable supply of PAHs in the influent, resulting in a constant formation of OPAHs. In another aspect, the retention time of activated sludge in the WWTP was 20-25 days. During this time, the formed AQ might combine with the DOMs, which would decrease the biodegradation rate of $A Q$.

In the real WWTP in Beijing, the removal efficiencies of the LMW PAHs from the aqueous phase were between $51 \%$ and $75 \%$, and the HMW PAHs between 7\% and 75\% [7]. In WWTPs of Sweden and Lithuania, the removal efficiencies of the LMW PAHs and MPAHs from the influent (aqueous phase) ranged from 33\% to $100 \%$, while those of the HMW compounds from $18 \%$ to $60 \%$ [15]. In the batch experiment, the removal efficiencies of different molecular weight PAHs in aqueous phase were relatively high and did not varied much (96-100\%). The differences might also be resulted from the association of the target molecules with DOMs in the influent and the returned activated sludge in real WWTPS.

From the temperature aspect, in the real WWTP, the removal efficiencies of the PAHs from the aqueous phase were between $7 \%$ and $71 \%$ in summer; while PAHs showed removal efficiencies of $42-75 \%$ in winter [7]. It was concluded in the real WWTP that PAHs were parted removed from the aqueous phase by adsorption but not only biodegradation, including the LMW compounds, due to the lower adsorption efficiency at the higher temperature. However, in the batch experiment (Fig. 3), the removal efficiencies of the LMW compounds in the LT condition were lower than the MT and HT condition before the first $48 \mathrm{~h}$, suggesting that after adsorption firstly, the biodegradation was the major removal process. The HMW compounds could not be totally biodegraded in the total phase, indicating that the HMW compounds were removed both by biodegradation and adsorption. The final removal efficiencies of PAHs were $100 \%$ in the aqueous phase of the batch experiment. The different results might indicate that the microorganism and the adsorption capacity of the sludge for PAH degradation was not enough for the PAHs loading in this real WWTP. However, it was demonstrated in Section 3.2.3 that microorganism and the adsorption capacity were sufficient for PAH removal. As a result, the different results should be influenced by the combination of DOMs with PAHs in the real WWTP.

The different initial concentrations did not influence the removal efficiencies, suggesting that the various concentrations of PAHs in normal municipal wastewater would not influence the removal efficiencies in the real WWTP. 


\section{Conclusion}

This study demonstrated the transformation from PAHs to OPAHs during the biological treatment process and the influence factors for PAHs and OPAHs biodegradation/biotransformation. The transformation from Ant to AQ during biological wastewater treatment process was valid though a batch experiment. A higher temperature $\left(25^{\circ} \mathrm{C} / 35^{\circ} \mathrm{C}\right)$ and initial concentration $(5.0 \mu \mathrm{g} / \mathrm{L})$ of Ant promoted the transformation process, compared with a lower temperature $\left(10^{\circ} \mathrm{C}\right)$ and initial concentration $(0.5 \mu \mathrm{g} / \mathrm{L})$. The relevant functional fungi for the transformation were detectable in the activated sludge. Furthermore, the possible factors influencing the biodegradation of PAHs and OPAHs were determined. The lower molecular weight compounds were more easily biodegraded than the higher ones. The temperature around $25^{\circ} \mathrm{C}$ was more appropriate for compounds removal than $35^{\circ} \mathrm{C}$ or $10^{\circ} \mathrm{C}$, because the microbial species were more plentiful in activated sludge at $25^{\circ} \mathrm{C}$. Different initial concentrations $(0.5 \mu \mathrm{g} / \mathrm{L}, 5.0 \mu \mathrm{g} / \mathrm{L})$ did not significantly influence the removal efficiencies, indicating the sufficiency of the degradation microorganism and the adsorption capacity of the sludge for PAHs in these initial concentrations. However, the compounds we added to the samples could be more easily removed than those inherent in the samples, probably resulting from the association of the compounds with the DOMs. Compared with the real wastewater treatment process, the degradation process of $\mathrm{AQ}$ and some influencing factors for PAHs and OPAHs removal were different in the batch experiment, which could be explained by the real environmental factors in WWTPs. Consequently, the results from the batch experiment could be valid indicators for the effect of real wastewater treatment process.

\section{Acknowledgements}

This work was supported by National Natural Science Foundation of China (Grant No. 51420105012, 51508552 and 51138006).

\section{Appendix A. Supplementary data}

Supplementary data associated with this article can be found, in the online version, at http://dx.doi.org/10.1016/j.cej.2016.04.139.

\section{References}

[1] M. Dimashki, S. Harrad, R.M. Harrison, Measurements of nitro-PAH in the atmospheres of two cities, Atmos. Environ. 34 (2000) 2459-2469.

[2] J.N. Pitts Jr., Nitration of gaseous polycyclic aromatic hydrocarbons in simulated and ambient urban atmospheres: a source of mutagenic nitroarenes, Atmos. Environ. (1967) 21 (1987) 2531-2547.

[3] J. Arey, B. Zielinska, R. Atkinson, A.M. Winer, T. Ramdahl, J.N. Pitts Jr., The formation of nitro-PAH from the gas-phase reactions of fluoranthene and pyrene with the $\mathrm{OH}$ radical in the presence of NOx, Atmos. Environ. 20 (1986) (1967) 2339-2345.

[4] S. Lundstedt, P.A. White, C.L. Lemieux, K.D. Lynes, L.B. Lambert, L. Oberg, P. Haglund, M. Tysklind, Sources, fate, and toxic hazards of oxygenated polycyclic aromatic hydrocarbons (PAHs) at PAH-contaminated sites, Ambio 36 (2007) 475-485.

[5] J.L. Durant, W.F. Busby Jr., A.L. Lafleur, B.W. Penman, C.L. Crespi, Human cell mutagenicity of oxygenated, nitrated and unsubstituted polycyclic aromatic hydrocarbons associated with urban aerosols, Mutat. Res. Genet. Toxicol. Environ. Mutagen. 371 (1996) 123-157.
[6] H. Tokiwa, Y. Ohnishi, H.S. Rosenkranz, Mutagenicity and carcinogenicity of nitroarenes and their sources in the environment, Crit. Rev. Toxicol. 17 (1986) 23-58.

[7] M. Qiao, W.X. Qi, H.J. Liu, J.H. Qu, Occurrence, behavior and removal of typical substituted and parent polycyclic aromatic hydrocarbons in a biological wastewater treatment plant, Water Res. 52 (2014) 11-19.

[8] M. Qiao, W.X. Qi, H.J. Liu, J.H. Qu, Oxygenated, nitrated, methyl and parent polycyclic aromatic hydrocarbons in rivers of Haihe River System, China: occurrence, possible formation, and source and fate in a water-shortage area, Sci. Total Environ. 481 (2014) 178-185.

[9] W.X. Qi, H.J. Liu, J.H. Qu, C.Z. Hu, H.C. Lan, M. Berg, H.M. Ren, W. Xu, Polycyclic aromatic hydrocarbons in effluents from wastewater treatment plants and receiving streams in Tianjin, China, Environ. Monit. Assess. 177 (2011) 467480.

[10] B.W. Bogan, R.T. Lamar, K.E. Hammel, Fluorene oxidation in vivo by Phanerochaete chrysosporium and in vitro during manganese peroxidasedependent lipid peroxidation, Appl. Environ. Microbiol. 62 (1996) 1788-1792.

[11] K.E. Hammel, B. Green, W.Z. Gai, Ring fission of anthracene by a eukaryote, Proc. Natl. Acad. Sci. 88 (1991) 10605-10608.

[12] B.R.M. Vyas, S. Bakowski, V. Šašek, M. Matucha, Degradation of anthracene by selected white rot fungi, FEMS Microbiol. Ecol. 14 (1994) 65-70.

[13] B.E. Andersson, T. Henrysson, Accumulation and degradation of dead-end metabolites during treatment of soil contaminated with polycyclic aromatic hydrocarbons with five strains of white-rot fungi, Appl. Microbiol. Biotechnol. 46 (1996) 647-652.

[14] C. Vogelsang, M. Grung, T.G. Jantsch, K.E. Tollefsen, H. Liltved, Occurrence and removal of selected organic micropollutants at mechanical, chemical and advanced wastewater treatment plants in Norway, Water Res. 40 (2006) 3559-3570.

[15] P.A. Bergqvist, L. Augulytė, V. Jurjonienė, PAH and PCB removal efficiencies in Umeå (Sweden) and Šiauliai (Lithuania) municipal wastewater treatment plants, Water Air Soil Pollut. 175 (2006) 291-303.

[16] W. Tian, J. Bai, K. Liu, H. Sun, Y. Zhao, Occurrence and removal of polycyclic aromatic hydrocarbons in the wastewater treatment process, Ecotoxicol. Environ. Saf. 82 (2012) 1-7

[17] T.T. Pham, S. Proulx, PCBs and PAHs in the Montreal Urban Community (Quebec, Canada) wastewater treatment plant and in the effluent plume in the St Lawrence River, Water Res. 31 (1997) 1887-1896.

[18] M. Qiao, W.X. Qi, H.J. Liu, J.H. Qu, Simultaneous determination of typical substituted and parent polycyclic aromatic hydrocarbons in water and solid matrix by gas chromatography-mass spectrometry, J. Chromatogr. A 1291 (2013) 129-136.

[19] U. Koljalg, K.H. Larsson, K. Abarenkov, R.H. Nilsson, I.J. Alexander, U. Eberhardt, S. Erland, K. Hoiland, R. Kjoller, E. Larsson, T. Pennanen, R. Sen, A.F.S. Taylor, L. Tedersoo, T. Vralstad, B.M. Ursing, UNITE: a database providing Web-based methods for the molecular identification of ectomycorrhizal fungi, New Phytol. 166 (2005) 1063-1068.

[20] Q. Wang, G.M. Garrity, J.M. Tiedje, J.R. Cole, Naive Bayesian classifier for rapid assignment of rRNA sequences into the new bacterial taxonomy, Appl, Environ. Microbiol. 73 (2007) 5261-5267.

[21] R. Meulenberg, H.H. Rijnaarts, H.J. Doddema, J.A. Field, Partially oxidized polycyclic aromatic hydrocarbons show an increased bioavailability and biodegradability, FEMS Microbiol. Lett. 152 (1997) 45-49.

[22] C.E. Cerniglia, Fungal metabolism of polycyclic aromatic hydrocarbons: past, present and future applications in bioremediation, J. Ind. Microbiol. Biotechnol. 19 (1997) 324-333.

[23] S. Oh, Q. Wang, W.S. Shin, D.I. Song, Effect of salting out on the desorptionresistance of polycyclic aromatic hydrocarbons (PAHs) in coastal sediment, Chem. Eng. J. 225 (2013) 84-92.

[24] X. Vecino, L. Rodriguez-Lopez, J.M. Cruz, A.B. Moldes, Sewage sludge polycyclic aromatic hydrocarbon (PAH) decontamination technique based on the utilization of a lipopeptide biosurfactant extracted from corn steep liquor, J. Agric. Food Chem. 63 (2015) 7143-7150.

[25] H. Yu, G.H. Huang, H.N. Xiao, L. Wang, W. Chen, Combined effects of DOM and biosurfactant enhanced biodegradation of polycylic armotic hydrocarbons (PAHs) in soil-water systems, Environ. Sci. Pollut. Res. 21 (2014) 1053610549.

[26] M. Aryal, M. Liakopoulou-Kyriakides, Biodegradation and kinetics of phenanthrene and pyrene in the presence of nonionic surfactants by Arthrobacter Strain Sphe3, Water Air Soil Pollut. 224 (2013) 1426-1436.

[27] J. Wu, Research on the Characteristics of Activated Sludge at Different Temperature, Tianjin University, 2005.

[28] H. Xu, Study on Assessment of Polycyclic Aromatic Hydrocarbons Bioavailability in Soil, Shandong Normal University, 2010 